\title{
Retrobulbar metastasis and intracranial invasion from postoperative hepatocellular carcinoma: A case report and review of the literature
}

\author{
CHUN-YONG CHEN ${ }^{1}$, JIAN-HONG ZHONG ${ }^{2}$ and JING-LI LIU ${ }^{1}$ \\ ${ }^{1}$ Department of Neurology, The First Affiliated Hospital; ${ }^{2}$ Department of Hepatobiliary Surgery, \\ The Affiliated Tumor Hospital, Guangxi Medical University, Nanning, Guangxi 530021, P.R. China
}

Received August 1, 2014; Accepted November 6, 2014

DOI: $10.3892 / 01.2014 .2733$

\begin{abstract}
Hepatocellular carcinoma (HCC) is the most common malignant cancer of the liver and the third ranking cause of cancer-related mortality worldwide. Following the diagnosis of HCC, intrahepatic and extrahepatic metastasis patients account for $\sim 50-75 \%$ of all HCC cases, lung and regional lymph nodes metastasis are the most common; metastasis to bone, skin and adrenal glands are rare, orbit metastasis and intracranial invasion are extremely rare. The present study reports the rare case of a patient with HCC that metastasized to the head. The patient presented with retrobulbar and intracranial invasion, and sub-scalp extension. Based on imaging findings, the lesion was initially misdiagnosed as meningioma, however, postoperative pathological examinations resulted in a definitive diagnosis of HCC metastasis. Based on the present case and a review of the relevant literature published since 2009 , the current study recommends that metastasis must be considered when diagnosing retrobulbar head lesions in patients with $\mathrm{HCC}$, regardless of contradictory imaging findings and other clinical indicators, which may closely mimic the original head lesion.
\end{abstract}

\section{Introduction}

Hepatocellular carcinoma (HCC) is the sixth most common type of cancer and third most common cause of cancer-related mortality worldwide. A total of $\sim 50,000$ new cases are estimated to occur annually (1). With an advanced diagnosis and effective early treatment via curative resection, ablation or transplantation, the median survival time of patients is

Correspondence to: Professor Jing-Li Liu, Department of Neurology, The First Affiliated Hospital, Guangxi Medical University, 6 Shuangyong Road, Nanning, Guangxi 530021, P.R. China

E-mail: 1ilicomet@163.com

Key words: hepatocellular carcinoma, retrobulbar metastasis, intracranial invasion, meningioma
$>5$ years (2). HCC is strongly associated with chronic hepatitis B virus (HBV) infection; just over half of HCC patients worldwide are $\mathrm{HBV}$-infected and the proportion is much higher in Asia (1). A total of $80 \%$ of HCC patients from Asia and South Africa present with risk factors including HBV and aflatoxin B1 exposure (2). Furthermore, a substantial proportion of HCC patients (range, 14-37\%) develop extrahepatic HCC metastasis (3). Such metastasis most commonly occurs in adjacent lymph nodes and the lungs, and less frequently in the bones, adrenal glands and brain (4). Surgical resection is a safe and effective strategy for the treatment of numerous patients with primary or metastatic $\mathrm{HCC}$, however, recurrence is common, with $\leq 70 \%$ of patients exhibiting tumor recurrence within five years of curative surgery (5). The present study describes an unusual case of a patient with chronic HBV infection and HCC who, five months following surgical resection, presented with a retrobulbar and intracranial lesion. Although the lesion mimicked meningioma, subsequent pathological analysis identified it as HCC metastasis. Additionally, the patient did not show any evidence of tumor recurrence in the liver. Written informed consent for the publication of this study was obtained from the patient.

\section{Case report}

A 43 year-old male was admitted to the Neurosurgery Department of the First Affiliated Hospital of Guangxi Medical University (Nanning, China) on the 8 April, 2014 with approximately a one-month history of mild headache and mild protopsis of the left eye. Upon physical examination, the patient exhibited mild protopsis of the left eye and ipsilateral visual acuity of 0.3 (normal range, 1.0-2.0) and contralateral visual acuity of 1.5 (normal range, 1.0-2.0). A frontal mass under the scalp adjacent to the left orbit was visible, however, no pulsation or other positive clinical manifestations were observed on palpation. Furthermore, the patient reported no abnormal sensations around the left eyeball, vomiting or vertigo.

Computed tomography (CT) and cerebral magnetic resonance imaging (MRI) scans identified retrobulbar metastasis and intracranial invasion with lytic changes in the left orbital and temporal bones (Fig. 1). Almost homogeneous isointensity was visible on T1- and T2-weighted images, as well 

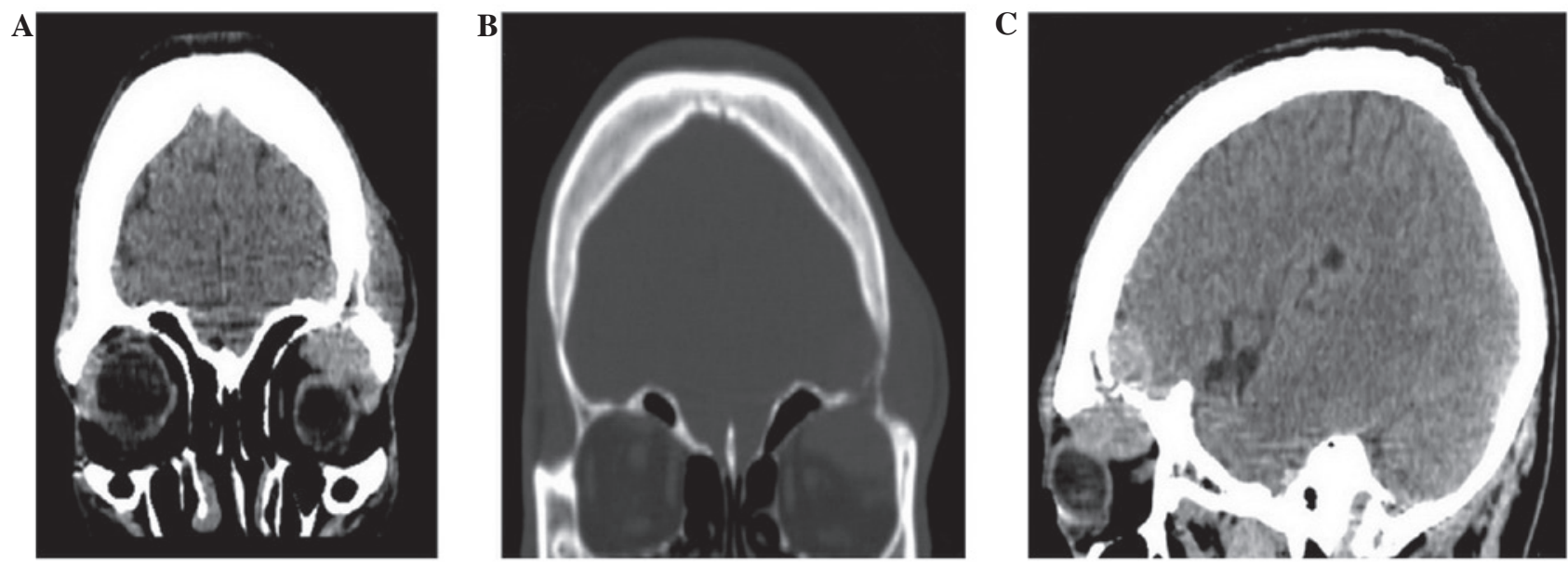

Figure 1. Computed tomography imaging of the patient, including: (A) A coronal plane image demonstrating intracranial and extracranial masses in the left orbit region under the scalp; (B) a coronal plane image indicating lytic changes in the skull bone; and (C) a sagittal plane image showing a cranio-orbital mass.
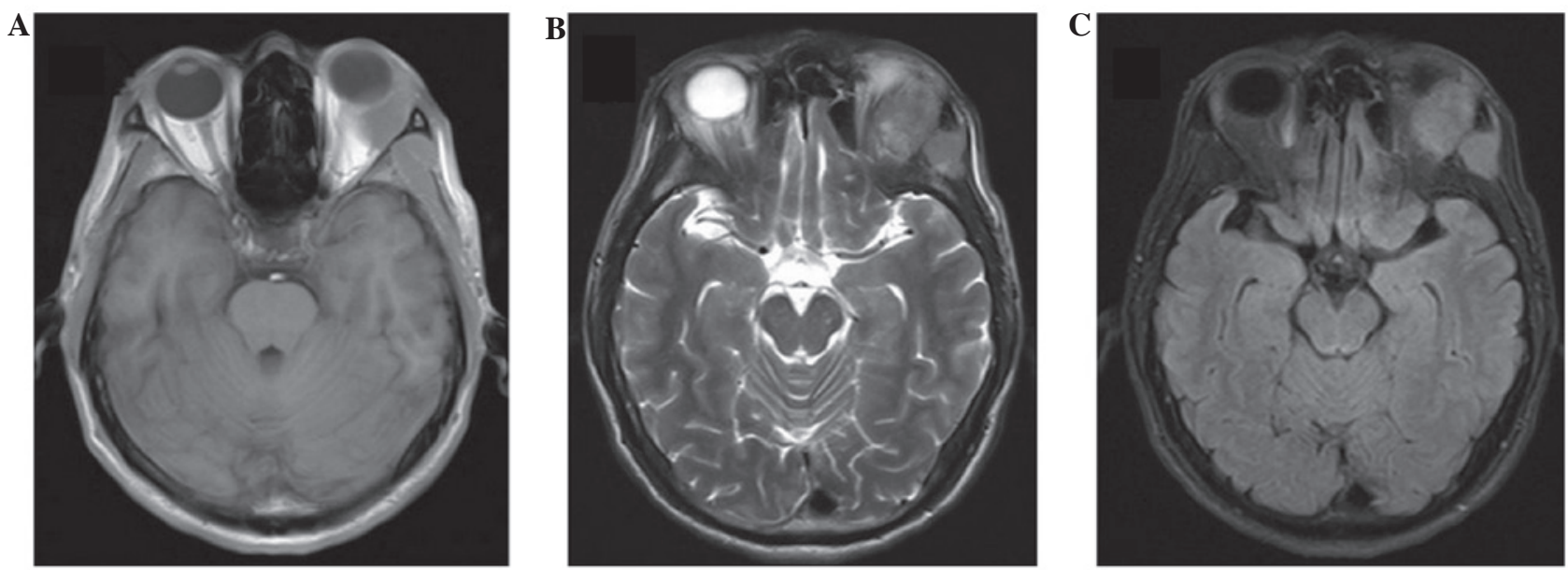

Figure 2. Magnetic resonance imaging of the patient. The isointensity of the lesion is visible on (A) T1-weighted, (B) T2-weighted and (C) fluid-attenuated inversion recovery images.

as on fluid-attenuated inversion recovery images (Fig. 2). Additionally, a dural tail sign with lytic changes in the adjacent skull bones was present on T1-enhanced MRI images (Fig. 3). Therefore, a diagnosis of meningioma with retrobulbar and under-scalp invasion was determined.

An enhanced CT scan of the upper abdomen revealed no recurrent hepatic lesions (Fig. 4), however, serological analysis demonstrated that the patient was positive for hepatitis B $(\mathrm{HB})$ surface antibody (Ab), $\mathrm{HB}$ e Ab and $\mathrm{HB}$ core Ab. Additionally, the patient exhibited an $\alpha$-fetoprotein level of $29.07 \mathrm{ng} / \mathrm{ml}$ (normal range, $0-11 \mathrm{ng} / \mathrm{ml}$ ), however, no abnormalities were detected in the liver biochemistry or in other routine tests.

Due to these findings, the intracranial mass was surgically resected. Following scalp incision, a round mass with the appearance of fish meat and a diameter of $5 \mathrm{~cm}$ was identified to have invaded the local skull area. It was tightly adhered to the dura mater and protruded into the left orbit. Pathological examination of the lesion following hematoxylin and eosin staining was used to identify the mass as HCC (Fig. 5). Therefore, the patient was definitively diagnosed with retrobulbar metastasis and intracranial invasion from postoperative hepatocellular carcinoma (HCC).
Table I. Location of hepatocellular carcinoma metastases to the head reported in the Chinese- and English-language literature since 2009.

\begin{tabular}{lc}
\hline Lesion location & $\mathrm{n}(\%)$ \\
\hline Orbit & $11(28.2)$ \\
Face & $8(20.5)$ \\
Skull base & $5(12.8)$ \\
Scalp & $4(10.3)$ \\
Meninges & $4(10.3)$ \\
Calvaria & $3(7.7)$ \\
Cerebrum & $2(5.1)$ \\
Dura and scalp & $2(5.1)$ \\
Total & $39(100)$ \\
\end{tabular}

\section{Discussion}

The combination of MRI imaging results and the absence of detectable $\mathrm{HCC}$ lesions on the liver by enhanced $\mathrm{CT}$ of the upper 

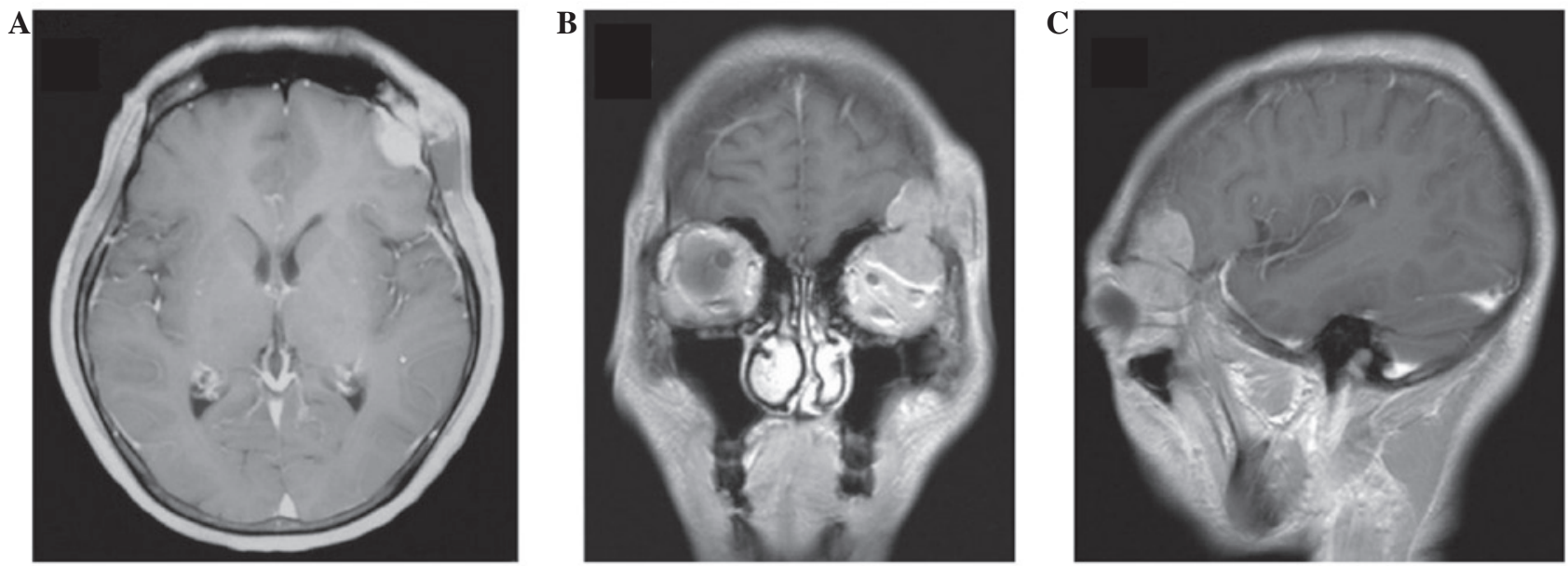

Figure 3. T1-enhanced magnetic resonance imaging of the patient revealed (A) dural attachment and a mild dural tail sign, and (B) coronal and (C) saggital plane images revealed a cranio-orbital invasive lesion with moderate enhancement.
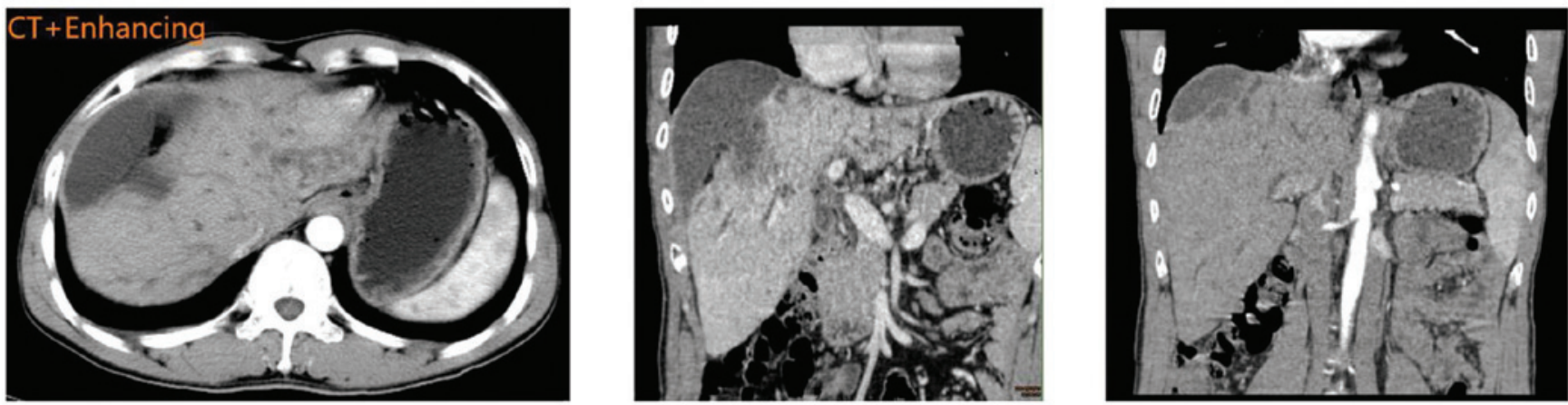

Figure 4. Multiple plane enhanced computed tomography of the upper abdomen revealed no recurrent hepatocellular carcinoma lesion in the liver.
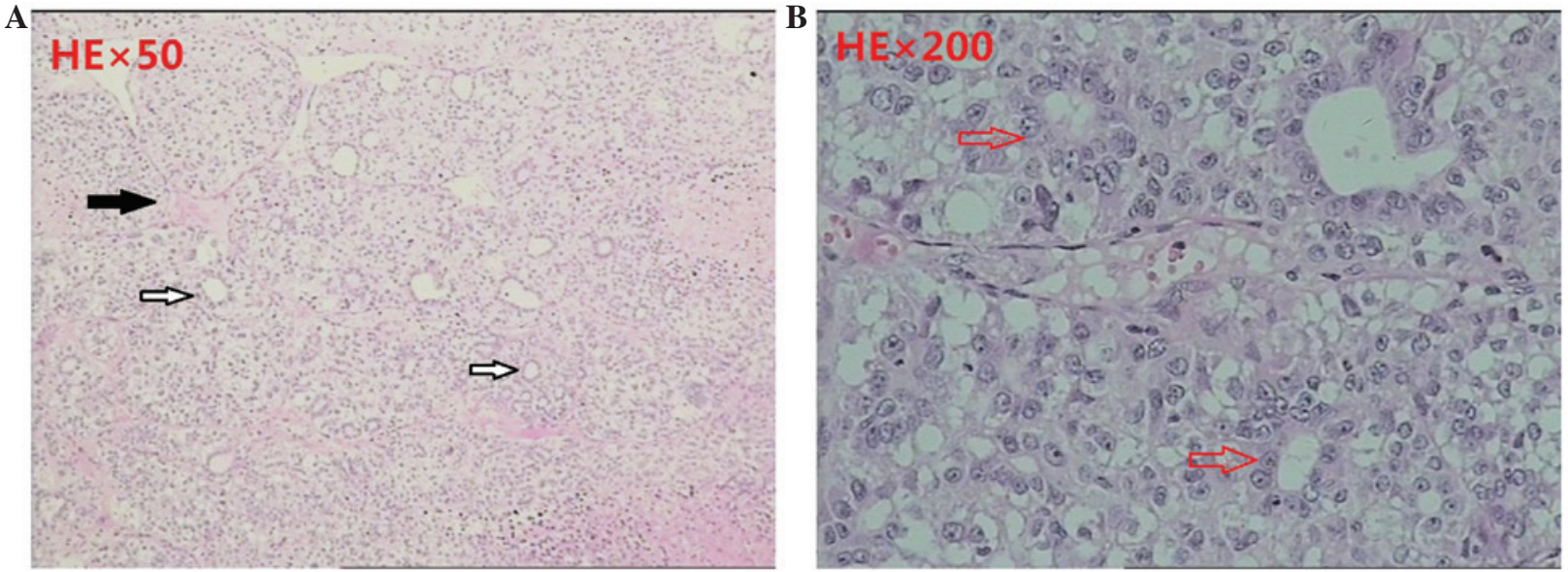

Figure 5. Postoperative pathology of the excised metastatic lesion, showing (A) the portal area (black arrow), bile ducts (white arrow; magnification, x50); and (B) nest-shaped cell clusters with central lumen that adopted the shape of the bile ducts (arrow; magnification, x200; hematoxylin and eosin staining).

abdomen resulted in an initial misdiagnosis of meningioma with retrobulbar invasion. Meningiomas are the second most common type of primary brain tumor, accounting for $15-20 \%$ of primary brain tumors; the intracranial mass protrudes extracranially via the bones and mimics thickened bone (6). Ultimately, surgical findings and postoperative pathology revealed the mass in the present patient to be a HCC metastatic lesion.
The current study conducted a review of the relevant Chinese- and English-language medical literature, and identified 39 cases of HCC metastasis to the head reported since 2009 (Table I) (4,7-35). Of these 39 patients, 11 exhibited peri-orbital metastasis similar to that of the present patient (Table II) $(4,17-19,25,30)$. Of the 11 cases with HCC metastasis to the orbit published since 2009, all were male (with 


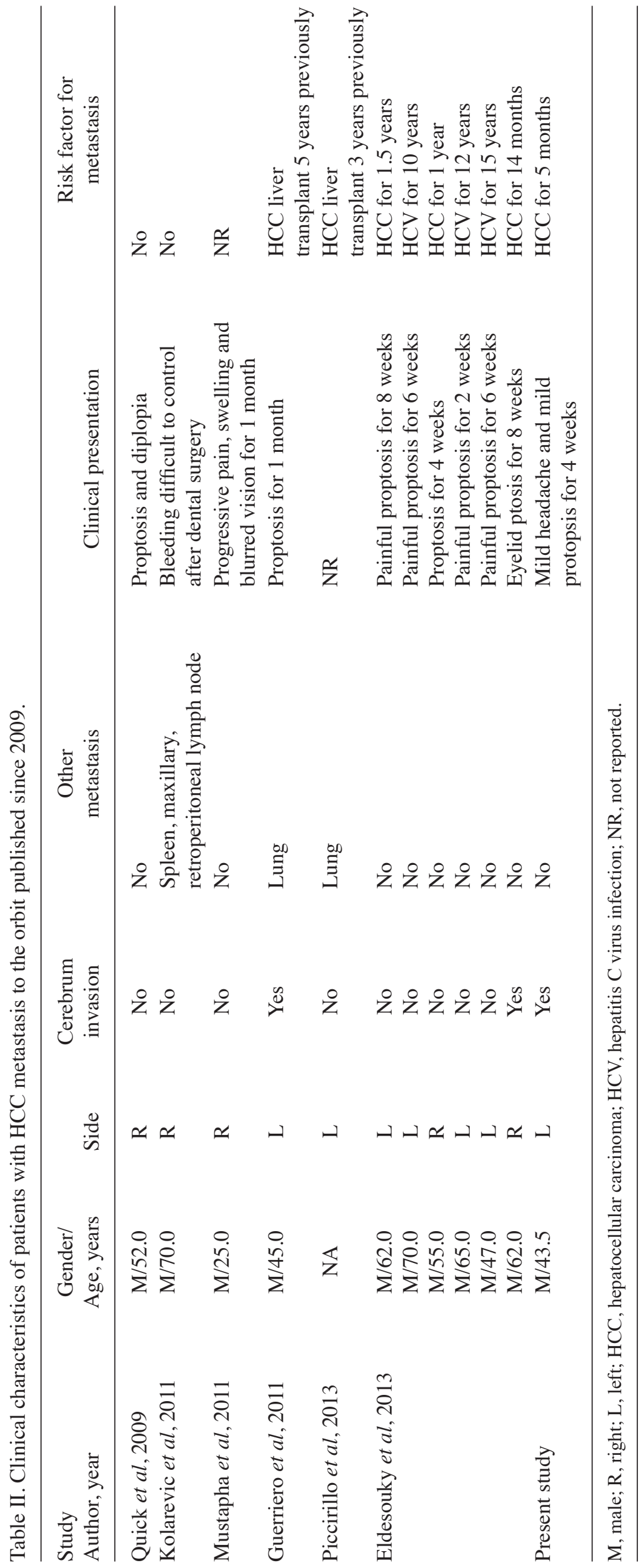


the exception of no gender being reported in one case)e, five exhibited invasion from the right side, only two patients demonstrated peri-orbital invasion combined with cerebral invasion, yet cerebral invasion also occurred in the present case. Painful proptosis was commo; seven patients suffered from this, as well as the patient in the present case. Two patients had not been diagnosed with HCC with metastasis when they were hospitalized. The metastatic mass invaded the cerebrum in only two cases and two patients experienced metastasis to the head five years following liver transplantation to treat HCC $(28,33)$. The first case was a 70-year-old male, who had HCC and underwent liver transplantation in August 2006, and was without HCC recurrence in the following 4 years. However, the patient was identified to have a $3-\mathrm{cm}$ painless, subcutaneous mass; CT scans showed an osteolytic lesion in the frontal bone without dural matter invasion, which was pathologically diagnosed as HCC metastasis. The second case was a 38-year-old male, who underwent surgery to remove a liver mass and received a liver transplantation 4 months previously; however, a well-defined localized painless mass was identified on the right temporal side of the patient's scalp. MRI showed that the mass involved the outer and inner skull tables, and was attached to the dura matter. Pathological analysis led to a diagnosis of HCC metastasis.

The present study illustrates that extrahepatic metastasis can occur with or without concurrent intrahepatic lesions following curative resection for HCC. Surgery is widely considered to be an effective treatment strategy for extrahepatic metastatic lesions from HCC; however, systemic sorafenib therapy may be another possible treatment modality, as it has demonstrated improved survival in HCC metastatic patients (36).

In conclusion, the present study highlights the importance of considering metastasis in the differential diagnosis of patients with a history of HCC who present with an intracranial mass. The possibility of HCC metastasis must be acknowledged despite the results of imaging procedures and other clinical indicators, as brain metastases may closely mimic meningioma.

\section{Acknowledgements}

The present study was supported by the National Natural Science Foundation of China (grant no. 81160168).

\section{References}

1. Montalto G, Cervello M, Giannitrapani L, Dantona F, Terranova A and Castagnetta LA: Epidemiology, risk factors, and natural history of hepatocellular carcinoma. Ann N Y Acad Sci 963: 13-20, 2002.

2. Forner A, Llovet JM and Bruix J: Hepatocellular carcinoma. Lancet 379: 1245-1255, 2012.

3. Katyal S, Oliver JH III, Peterson MS, Ferris JV, Carr BS and Baron RL: Extrahepatic metastases of hepatocellular carcinoma. Radiology 216: 698-703, 2000.

4. Quick AM, Bloomston M, Kim EY, Hall NC and Mayr NA: Complete response to radiation therapy of orbital metastasis from hepatocellular carcinoma. World J Gastroenterol 15: 6000-6003, 2009

5. European Association for the Study of the Liver; European Organization for Research and Treatment of Cancer: EASL-EORTC clinical practice guidelines: management of hepatocellular carcinoma. J Hepatol 56: 908-943, 2012.
6. Jang SY, Kim CH, Cheong JH and Kim JM: Extracranial extension of intracranial atypical meningioma en plaque with osteoblastic change of the skull. J Korean Neurosurg Soc 55: 205-207, 2014.

7. Tateno H, Hoshino T, Takahashi K, Matsumura M, Sakaida N and Ohe C: A rapidly progressed metastatic choroidal tumor from a hepatocellular carcinoma. Nihon Ganka Gakkai Zasshi 113: 107-111, 2009 (In Japanese).

8. Trivedi P, Gupta A, Pasricha S, Agrawal G and Shah M: Isolated skull base metastasis as the first manifestation of hepatocellular carcinoma - a rare case report with review of literature. J Gastrointest Cancer 40: 10-14, 2009.

9. Pallini R, Sabatino G, Doglietto F, Lauretti L, Fernandez E and Maira G: Clivus metastases: report of seven patients and literature review. Acta Neurochir (Wien) 151: 291-296, 2009.

10. Somerset H, Witt JP and Kleinschmidt-Demasters BK Hepatocellular carcinoma metastases to the epidural space. Arch Pathol Lab Med 133: 1975-1980, 2009.

11. Ma XH: Hepatocellular carcinoma metastasis to brain: A case report. Chinese Hepatology 14: 336, 2009.

12. Vitale AR, Compilato D, Coletti G, et al: Metastatic hepatocellular carcinoma of the parotid region without lung metastasis: a case report. Int J Oral Maxillofac Surg 38: 696-698, 2009.

13. Kim BG, Yoon SM, Bae HG and Yun IG: Spontaneous intracranial epidural hematoma originating from dural metastasis of hepatocellular carcinoma. J Korean Neurosurg Soc 48: 166-169, 2010.

14. Woo KM, Kim BC, Cho KT and Kim EJ: Spontaneous epidural hematoma from skull base metastasis of hepatocellular carcinoma. J Korean Neurosurg Soc 47: 461-463, 2010.

15. Bair MJ, Lei WY and Chen CL: Electronic images of the month. An unusual presentation of hematemesis: a presentation of maxillary metastasis from hepatocellular carcinoma. Clin Gastroenterol Hepatol 8: e61-e62, 2010.

16. Saneluxsana $S$ and Urathamakul S: Recurrent skull metastasis of hepatocellular carcinoma at 2 month post operation. J Med Assoc Thai 93 (Suppl 6): S212-S214, 2010.

17. Kolarević D, Tomasević Z, Boricić I, et al: Metastasis of hepatocellular carcinoma presented as a tumor of the maxillary sinus and retrobulbar tumor. Vojnosanit Pregl 68: 359-362, 2011.

18. Mustapha SK and Madachi DA: Orbital metastasis of hepatocellular carcinoma: a case report. West Afr J Med 30: 305-307, 2011.

19. Guerriero S, Infante G, Giancipoli E, et al: Hepatocellular carcinoma metastasis to the orbit in a coinfected HIV+ HBV+ patient previously treated with orthotopic liver transplantation: A case report. Case Rep Ophthalmol Med 2011: 549270, 2011.

20. Walter CM, Kirby EJ, Vasconez HC and Rinker BD: Hepatocellular carcinoma metastatic to the scalp. J Craniofac Surg 22: 720-721, 2011.

21. Kato Y, Kikuchi N, Nishibu A, Ohtsuka M and Yamamoto T: Letter: Two cases of metastases to the scalp bone mimicking epidermoid cyst. Dermatol Online J 17: 13, 2011.

22. Lasiter JC, Liess BD, Zitsch RP III and Wieberg J: An expansile mandibular mass as the initial manifestation of hepatocellular carcinoma. Ear Nose Throat J 90: E19, 2011.

23. Yang JI, Kang JM, Byun HJ, et al: Metastatic hepatocellular carcinoma presenting as facial nerve palsy and facial pain. Korean J Hepatol 17: 319-322, 2011

24. Sanchez-Delgado J, Calzado S, de Haro C, et al: Long survival after resection of cranial metastases from hepatocellular carcinoma. Case report and review of the literature. Gastroenterol Hepatol 35: 12-16, 2012 (In Spanish)

25. Eldesouky MA, Elbakary MA, Shalaby OE and Shareef MM: Orbital metastasis from hepatocellular carcinoma: report of 6 cases. Ophthal Plast Reconstr Surg 30: e78-e82, 2014.

26. Pirbhai A, Kahawita S, Davis G, Dodd T, Thomas J and Selva D: Hepatocellular carcinoma metastasis to sphenoid wing. Orbit 32: 132-133, 2013.

27. Nemetz U, Tomazic PV, Walch C, et al: Pyramidal apex metastasis as primary manifestation of hepatocellular carcinoma. Otol Neurotol 34: e30-e31, 2013.

28. Turan I, Yapali S, Ozutemiz O and Karasu Z: Frontal skull metastasis extending through the scalp: initial sign of hepatocellular carcinoma recurrence 5 years after liver transplantation. Transplantation 95: e15-e16, 2013.

29. Miller ME, McCall AA, Juillard GF, Nadelman CM, Wang MB and Nabili V: Hepatocellular carcinoma metastatic to the mandible. Ear Nose Throat J 92: E17-E19, 2013.

30. Piccirillo M, Granata V, Albino V, et al: Can hepatocellular carcinoma (HCC) produce unconventional metastases? Four cases of extrahepatic HCC. Tumori 99: e19-e23, 2013. 
31. Tomanovic N, Krstic A, Brasanac D, Dimitrijevic M, Terzic T and Boricic I: Zygomatic bone metastasis as an initial presentation of hepatocellular carcinoma. Arch Iran Med 16: 675-678, 2013.

32. Brunetti AE, Popescu O and Silvestris N: Synchronous mandibular and giant parieto-occipital skull metastasis from hepatocellular carcinoma. Clin Gastroenterol Hepatol 11: xxvi, 2013.

33. Azarpira N, Dehghanian A, Safarian A and Kazemi K: Case report of skull metastasis from hepatocellular carcinoma after a liver transplant. Exp Clin Transplant 12: 265-268, 2014.
34. Pan Z, Yang G, Yuan T, et al: Leptomeningeal metastasis from hepatocellular carcinoma with other unusual metastases: a case report. BMC Cancer 14: 399, 2014.

35. Guo X, Yin J and Jiang Y: Solitary skull metastasis as the first symptom of hepatocellular carcinoma: case report and literature review. Neuropsychiatr Dis Treat 10: 681-686, 2014.

36. Chua TC and Morris DL: Exploring the role of resection of extrahepatic metastases from hepatocellular carcinoma. Surg Oncol 21: 95-101, 2012. 